\title{
PRODUCTS OF NORMAL SEMI-FIELDS
}

\author{
BY \\ ALBERT NEUHAUS
}

1. Introduction. The normal rings considered by Teichmüller $\left(^{1}\right)$ are a generalization of normal fields. They are commutative semi-simple algebras with a group of automorphisms whose order is the order of the algebra. Since they are direct sums of isomorphic fields, Albert $\left({ }^{2}\right)$ called these normal rings semifields. In case the group is cyclic he called them cyclic semi-fields.

Albert considered the direct product of two cyclic semi-fields 3 and $\mathfrak{Y}$ of order $n$ over the reference field. If $[G]$ and $[H]$ are the defining cyclic automorphism groups of $\mathfrak{Z}$ and $\mathfrak{Y}$ respectively he defined the sub-algebra $\mathfrak{W}_{k}$ of all quantities of $3 \times \mathfrak{V}$ unaltered by $\left[G H^{k}\right]$, for any integer $k$, and proved

$$
\mathfrak{B} \times \mathfrak{Y}=\mathfrak{3} \times \mathfrak{B}_{k} \text {. }
$$

These factorizations of $3 \times \mathfrak{Y}$ may be thought of as being determined by the factorizations

$$
[G] \times[H]=\left[G H^{k}\right] \times[H]
$$

of the group $[G] \times[H]$.

In the present discussion we shall consider normal semi-fields $\mathfrak{S}$ with an arbitrary group (\$). We shall show that we can obtain other direct factorizations of $\mathfrak{S} \times \mathfrak{T}$ with group $\left(\mathfrak{S} \times \mathbb{S}^{*}\right.$ if $\mathbb{B}$ contains a normal divisor $\mathfrak{N}$, such that $\left(\mathfrak{S} / \mathfrak{N}\right.$ is equivalent to a subgroup $\mathfrak{S}_{c}^{*}$ of the centrum of $\mathfrak{S}^{*}$, the group of $\mathfrak{T}$. We shall generalize Albert's cyclic systems to normal systems (ङ્, (S). Then we shall see that the set $\Sigma$ of all normal systems with group $B$ with the property

$$
\mathfrak{S} / \mathfrak{N} \cong \mathfrak{S}_{c}
$$

$\left(\mathfrak{S}_{c}\right.$ a subgroup of the centrum of $(\mathfrak{S})$ is closed under multiplication, and that this multiplication is associative if $\mathbb{S}$ is the direct product of $\mathfrak{N}$ and $\mathfrak{S}_{c}$. Finally $\Sigma$ is an abelian group if $B$ is abelian.

Furthermore as in the case of cyclic systems we shall consider crossed products of normal systems with their groups, and shall derive a connection between the properties of direct products of two such crossed products and the corresponding properties of normal systems.

Presented to the Society, September 8, 1939; received by the editors April 15, 1940. The author wishes to take this opportunity to express his gratitude to Professor A. A. Albert for his helpful criticism and guidance during the writing of this paper.

(1) Deutsche Mathematik, vol. 1 (1936), pp. 92-102, 192-238.

(2) Albert, A. A., Annals of Mathematics, (2), vol. 39 (1938), pp. 669-682, and Structure of Algebras, American Mathematical Society Colloquium Publications, vol. 24, 1939. 
2. Normal semi-fields. A separable semi-field $\subseteq$ of order $n$ over the reference field $\Omega$ is known $\left({ }^{2}\right)$ to be the direct sum of $t$ separable, isomorphic fields $\mathfrak{Q}_{\mu}$ of degree $s$ over $\Omega$, that is,

$$
\mathfrak{S}=\mathfrak{Q}_{1} \oplus \mathfrak{Q}_{2} \oplus \cdots \oplus \mathfrak{Q}_{t} \quad(s t=n) .
$$

We make the

Definition. Let $\mathfrak{S}$ be a separable semi-field of order $n$ over $\Omega$ and let $(\mathfrak{S})=\left(G_{1}, G_{2}, \cdots, G_{n}\right)$ be a group of automorphisms of $\mathfrak{S}$. Then we call $\mathfrak{S}$ a normal semi-field with automorphism group $\&$ if $\mathfrak{S}_{\mathbb{R}}=\left(e^{G_{1}}, e^{G_{2}}, \ldots, e^{G_{n}}\right)$ over $\mathfrak{R}$ for a field $\mathfrak{R}$ over $\Omega$ and pairwise orthogonal idempotents $e^{G_{\nu}}$.

This is exactly the definition of a normal ring as given by Teichmüller $\left({ }^{3}\right)$. Diagonal algebras are trivially normal semi-fields and normal fields also are normal semi-fields.

A normal semi-field $\mathbb{S}$ with group $\left(\mathbb{S}\right.$ is $\mathbb{B}$-irreducible $\left({ }^{4}\right)$, for the diagonal algebra $\widetilde{S}_{\mathfrak{l}}$ is irreducible since $\mathbb{S}$ is transitive with respect to the $e^{G_{\nu}}$. From Albert's lemma $\left.{ }^{4}\right)$ it follows that $\mathfrak{S}=\mathfrak{Q} \times\left(\mathbb{E}\right.$ with $\mathbb{E}=\left(e^{\sigma_{1}}, e^{\sigma_{2}}, \cdots, e^{\sigma_{t}}\right)$ for a set of $g_{\mu}$ of $\left(\xi\right.$. Teichmüller proved( $\left(^{5}\right)$ that $\mathscr{Q}$ is a normal field over $\Re$, whose automorphism group is the subgroup $\mathfrak{S}$ of $\mathbb{B}$ which leaves the elements of $\mathbb{E}$ unaltered.

We prove a result which, for the case where $\subseteq$ is a field, is a part of the well-known fundamental theorem of the Galois theory $\left({ }^{6}\right)$.

THEOREM 1. To every normal divisor $\mathfrak{R}$ of $\&$ corresponds a normal subsemi-field $\mathfrak{S}(\mathfrak{N})$ of $\mathfrak{S}$ consisting of all elements of $\mathfrak{S}$ unaltered by the automorphisms of $\mathfrak{N}$, and having $\mathbb{H} / \mathfrak{N}$ as a group. The order of $\mathfrak{S}(\mathfrak{N})$ is the order of $\mathbb{H} / \mathfrak{N}$.

For $\mathfrak{S}(\mathfrak{N})$ is a sub-algebra of a commutative semi-simple algebra and is therefore also commutative and semi-simple, hence $\mathfrak{S}(\mathfrak{N})$ is a semi-field. The group $\mathbb{H} / \mathfrak{N}$ is a group of automorphisms of $\mathfrak{S}(\mathfrak{N})$ since all elements of a coset of $\mathfrak{N}$ induce the same automorphism in $\mathfrak{S}(\mathfrak{R})$. It is normal since the scalar extension $\mathfrak{S}(\mathfrak{R})_{\mathfrak{R}}$ is the diagonal sub-algebra of $\mathfrak{S}_{\mathfrak{R}}$ unaltered by $\mathfrak{N}$ and $\mathfrak{S}(\mathfrak{N})_{\mathfrak{R}}$ has $\left(e^{n_{1}}+e^{n_{2}}+\cdots+e^{n_{i}}\right)^{g_{\mu}}(\mu=1, \cdots, j), i j=n$, as a basis, with $\mathfrak{N}=\left(n_{1}, n_{2}, \cdots, n_{i}\right), \quad \mathfrak{S}=g_{1} \mathfrak{N}+g_{2} \mathfrak{N}+\cdots+g_{j} \mathfrak{N}$.

Teichmüller proved this theorem in a different, more difficult way $\left({ }^{7}\right)$.

The converse, that to every normal semi-field of order $j$ of $\subseteq$ corresponds a normal divisor of order $i$ of $\$ \$$, does not hold in general as the following example shows. Let $\subseteq$ be diagonal with $(\$)$, the non-abelian group of order six, as an automorphism group. Then (S) consists of the elements $i, s, t, t^{2}, s t, s t^{2}$

(3) Deuring, M., Algebren, 1935, p. 96.

(4) Albert, A. A., Structure of Algebras, p. 78.

(5) Teichmüller, p. 98.

(6) Albert, A. A., Modern Higher Algebra, 1937, p. 174.

(7) Teichmüller, p. 203. 
where $i$ is the identity, $s$ and $t$ of orders two and three respectively, and $t s=s t^{2}$. Then $\subseteq$ has a normal basis $e^{i}, e^{s}, e^{t}, e^{t^{2}}, e^{s t}, e^{s t^{2}}$ of orthogonal idempotents. The diagonal algebra $\mathfrak{T}$ with $\left(e^{i}+e^{s}\right),\left(e^{t}+e^{s t}\right),\left(e^{t^{2}}+e^{s t^{2}}\right)$ as basis is a normal sub-semi-field with $\left(i, t, t^{2}\right)$ as automorphism group. But (B) does not have a normal divisor of order two.

Theorem 2. If $(S)$ is the direct product of two subgroups $\mathfrak{N}$, $\mathfrak{E}$, then $\mathfrak{S}$ is the direct product of their corresponding normal sub-semi-fields.

For, if $\mathfrak{S}=\mathfrak{N} \times \mathfrak{S}$ the $\mathfrak{N}$ and $\mathfrak{S}$ are normal divisors of $\mathfrak{G}$. If $n, i, j$ are the orders of $\mathfrak{B}, \mathfrak{N}, \mathfrak{S}$ respectively, then $n=i j$. Now $\mathfrak{S}(\mathfrak{N})$ has order $j$, $\mathfrak{S}(\mathfrak{S})$ order $i$, hence $\mathfrak{S}(\mathfrak{N}) \mathfrak{S}(\mathfrak{S})$ has order less than $n$ if and only if $\mathfrak{S}(\mathfrak{N})$ and $\mathfrak{S}(\mathfrak{S})$ have elements other than those of the reference field $\Omega$ in common. But such elements are elements of $\subseteq$ unaltered by all automorphisms of $\mathfrak{R}$ and $\mathfrak{S}$, hence of $\mathfrak{S}$, and they lie in $\Re$; $\mathfrak{S}(\mathfrak{N}) \mathfrak{S}(\mathfrak{S})$ has order $n$ and is equal to $\mathfrak{S}(\mathfrak{N}) \times \mathfrak{S}(\mathfrak{S})$ $=\mathfrak{s}$.

3. Factorizations of direct products of groups. As we indicated in the introduction, Albert considered direct factorizations of direct products of cyclic semi-fields determined by factorizations of the direct products of their groups. In our later considerations of the direct products we shall generalize the cyclic case by considering subsets of direct products of normal semi-fields unaltered by a subgroup $\mathscr{S}_{0}$ of $\mathbb{B S} \times \mathcal{S F}^{*}$, the direct product of their groups such that (S) $\times\left(5^{*}=\mathrm{S}_{0} \times()^{*}\right.$. Before doing so we wish to know about the possibility of such factorizations of the direct products of two groups. The required result is given by

TheOREM 3. Let (S) and (S* be any two finite groups. Then there exist fac-

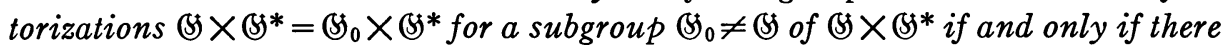
exists a normal divisor $\mathfrak{N}$ of $\mathbb{B}$ such that $\mathfrak{H} / \mathfrak{N}$ is equivalent to a subgroup $\mathfrak{S}_{c}^{*}$ of the centrum of $\mathbb{S}^{*}$. The group $\mathfrak{S}_{0}$ is equivalent to $\mathbb{S}$ and is contained in $\$ \mathfrak{S}_{c}^{*}$.

For, since $\mathbb{S}_{0}$ is a subgroup of $(B \times B *$ its elements must be products of elements in $(S)$ with elements in ()$^{*}$,

$$
g_{0}=g h^{*} \quad\left(g_{0} \text { in } \xi_{0}, g \text { in }(\$), h^{*} \text { in }\left(S^{*}\right) ;\right.
$$

on the other hand every element $g_{0}$ must be commutative with every element $g^{*}$ of $\left(3^{*}\right.$,

$$
g^{*} g_{0}=g_{0} g^{*}
$$

and

$$
g^{*} g h^{*}=g h^{*} g^{*}
$$

Since the elements of $(S)$ are commutative with those of $B^{*}$, we have

$$
g g^{*} h^{*}=g h^{*} g^{*},
$$


hence

$$
g^{*} h^{*}=h^{*} g^{*} \quad \text { for every } g^{*} \text { of }()^{*} .
$$

It follows that $h^{*}$ is in the centrum of $(5 *$.

Let $g_{\nu}(\nu=1,2, \cdots, m)$ be the elements of $(S)$, and $c_{\mu}{ }^{*}$ be elements of the centrum of $\mathbb{B}^{*}$. Then we know that every $g_{0}$ of $\mathbb{S}_{0}$ is uniquely expressible as the product of an element $g$ of $\$$ and an element $c^{*}$ of the centrum of $\left(S^{*}\right.$, so that

$$
g_{0} \equiv g c_{0}^{*}=c_{o}^{*} g .
$$

It follows that $\mathfrak{S}_{0}$ is equivalent to $\mathbb{S}$ under the correspondence $g_{0} \leftrightarrow g$. Since $\mathscr{B}_{0}$ is a group, we have then

$$
g_{0} h_{0}=g h c_{g}^{*} c_{h}^{*}=(g h)_{0}=(g h) c_{g h}^{*} .
$$

This holds if and only if $c_{g}^{*} c_{h}^{*}=c_{g h}^{*}$. Now it follows that there must exist a homomorphism

$$
g \rightarrow c_{0}^{*} \text {. }
$$

Hence these $c_{g}^{*}$ must form a group $\mathfrak{S}_{c}^{*}$ which, since the $c_{g}^{*}$ are in the centrum of $\mathbb{B}^{*}$, is a subgroup of this centrum. Thus $B$ is homomorphic to a subgroup $\mathfrak{S}_{c}^{*}$ of the centrum of $\mathfrak{S}^{*}$. Consequently $\left.{ }^{8}\right)$, there exists a normal divisor $\mathfrak{R}$ of $\mathfrak{B}$, such that $\mathbb{S} / \mathfrak{N}$ is equivalent to $\mathfrak{S}_{c}^{*}, \mathfrak{S} / \mathfrak{N} \cong \mathfrak{S}_{c}^{*}$.

Conversely, if $\mathbb{S}$ has a normal divisor $\mathfrak{N}$ such that $\mathbb{S} / \mathfrak{N}$ is equivalent to a subgroup $\mathfrak{S}_{c}^{*}$ of the centrum of $\mathfrak{S F}^{*}$, that is, if $\mathfrak{S}=g_{1} \mathfrak{N}+g_{2} \mathfrak{N}+\cdots+g_{j} \mathfrak{N}$ and $\mathfrak{S}_{c}^{*}=\left(c_{1}^{*}, c_{2}^{*}, \cdots, c_{j}^{*}\right)$, there is a one-one correspondence $g \mathfrak{R} \leftrightarrow c_{0}^{*}$ and hence a homomorphism between $\mathbb{F}$ and $\mathfrak{S}_{c}^{*}$, that is, if $\mathfrak{N}=\left(n_{1}, \cdots, n_{i}\right), i j=m$, $n_{\nu} g_{\mu} \rightarrow c_{\mu}^{*}$. We define

$$
g_{0 \nu, \mu}=n_{\nu} g_{\mu} c_{\mu}^{*} \quad(\nu=1, \cdots, i ; \mu=1, \cdots, j) .
$$

These $g_{0}$ form a group $\mathfrak{S}_{0}$. For, since $\mathfrak{N}$ is a normal divisor of the group $\mathbb{B}$ we have

$$
g n^{\prime}=n^{\prime \prime} g, \quad g_{0} h_{0}=n g c_{0}^{*} n^{\prime} h c_{h}^{*}=n n^{\prime \prime} g h c_{0}^{*} c_{h}^{*}
$$

and

$$
n n^{\prime \prime} g h \rightarrow c_{g}^{*} c_{h}^{*}
$$

But $\mathbb{S} / \mathfrak{N}$ is a group and there exists in $\mathfrak{N}$ an element $n^{\prime}$ such that

$$
\begin{gathered}
n g n^{\prime} h=n_{1} g_{1} \quad \text { (the unity of (S), } \\
g_{0}^{-1}=\left(n g c_{o}^{*}\right)^{-1}=n^{\prime} h c_{h}^{*}=h_{0} .
\end{gathered}
$$

The elements of $\mathscr{S}_{0}$ are commutative with the elements of $\mathfrak{S}^{*}$, and $\mathfrak{S}_{0}$ and $\mathfrak{S S}^{*}$

(8) van der Waerden, B. L. Moderne Algebra, vol. 1, 1930, p. 35. 
have only the unity element in common, hence

$$
\text { (B) } \times \mathbb{B}^{*}=\mathfrak{B S}_{0} \times \mathfrak{B F}^{*}
$$

and $\mathfrak{B}_{0} \leqq\left(S \mathfrak{S}_{c}^{*}\right.$. This proves our theorem.

4. Direct products. In our discussion of direct products of normal semifields we shall use

Theorem 4. A normal semi-field $\subseteq$ with group $\$=\left(G_{\mu} ; \mu=1, \cdots, n\right)$ has a normal basis

$$
\mathfrak{S}=\left(u^{G_{1}}, u^{G_{2}}, \cdots, u^{G_{n}}\right)
$$

over $\Omega$.

For $\mathfrak{S}=\mathfrak{Q} \times \mathbb{F}$ with $\mathfrak{Q}$ a normal field of degree $s$ over $\mathfrak{\Omega}$ and $\mathbb{E}$ a diagonal algebra of order $t$ over $\Omega, s t=n$. It is well-known $\left({ }^{9}\right)$ that $\mathfrak{Q}$ has a normal basis

$$
\xi^{h_{1}}, \xi^{h_{2}}, \cdots, \xi^{h_{s}}
$$

with $\xi$ in $\mathfrak{Q}$ and the $h_{\mu}$ the elements of the automorphism group $\mathfrak{S}$ of $\mathfrak{Q}$. On the other hand $\mathfrak{S}$ is the subgroup of $(\mathcal{S}$ whose elements leave the elements of $\mathscr{E}$ unaltered as we noted in $\$ 2$. There we saw that $\$$ has a basis

$$
e^{o_{1}}, e^{o 2}, \cdots, e^{o_{t}}
$$

of orthogonal idempotents, with $e$ in $₹$ and the $g_{\nu}$ a set of $t$ elements of $(B)$. Hence the $n$ elements $e^{g_{\mu}} \xi^{h_{\nu}}(\mu=1, \cdots, t ; \nu=1, \cdots, s)$ of $\mathfrak{S}$ form a basis of $\mathfrak{S}$.

Define $u=e^{\sigma_{1}} \xi^{h_{1}}$. Then for any element $G_{\sigma}$ of $(S)$ the quantity $u^{G_{\sigma}}$ is a certain $e^{\theta} \xi^{h}$ in $e^{\theta} \mathfrak{D}$. By taking all $n$ different $G$ of $(S)$ we get all $n$ elements $e^{o} \xi^{h}$. Thus $u=e^{g_{1}} \xi^{h_{1}}$ and its conjugates form a normal basis of $\mathfrak{S}$.

We now let $\mathfrak{\Im}=\mathfrak{S} \times \mathfrak{T}$ where $\mathfrak{S}$ and $\mathfrak{T}$ are normal semi-fields of order $m$ and $n$, and with groups $\&$ and $(\$)$ respectively, and prove

THEOREM 5. The direct product $\mathfrak{\Im}=\mathfrak{S} \times \mathfrak{T}$ is a normal semi-field of order $m n$ with group $(3 \times(3 *$.

For, since $\mathfrak{S}$ and $\mathfrak{T}$ are normal semi-fields there exist two fields $\mathfrak{R}^{\prime}$ and $\mathfrak{R}^{\prime \prime}$ over $\Re$, such that $\mathfrak{S}_{\mathfrak{R}^{\prime}}=\left(e^{G_{1}}, e^{G_{2}}, \cdots, e^{G_{m}}\right)$ and $\mathfrak{I}_{\mathfrak{R}^{\prime \prime}}=\left(f^{G_{1}^{*}}, f^{G_{2}^{*}}, \cdots, f^{G_{n}^{*}}\right)$ over $\mathfrak{R}^{\prime}$ and $\mathfrak{R}^{\prime \prime}$ respectively. Let $\mathfrak{R}$ be a composite of $\mathfrak{R}^{\prime}$ and $\mathfrak{R}^{\prime \prime}$ over $\mathfrak{\Omega}$. Then since $\mathfrak{R}$ contains $\ell^{\prime}$ and a field equivalent to $\mathfrak{R}^{\prime \prime}$ we have

$$
\mathfrak{S}_{\mathfrak{R}}=\left(e^{G_{1}}, e^{G_{2}}, \cdots, e^{G_{m}}\right), \mathfrak{T}_{\mathbb{R}}=\left(f^{G_{1}^{*}}, f^{G_{2}^{*}}, \cdots, f^{G_{n}^{*}}\right)
$$

and $\mathfrak{S}_{\mathfrak{R}}=\Im_{\mathfrak{R}} \times \mathfrak{T}_{\mathfrak{R}}=\left(e^{G_{1} f^{G_{1}^{*}}}, \cdots, e^{G_{1}} f_{G_{n}^{*}}, e^{G_{2}} f_{G_{1}^{*}}, \ldots, e^{G_{m} G_{n}^{*}}\right)$ over $\mathfrak{R}$. But $G_{\mu} G_{\nu}{ }^{*}(\mu=1, \cdots, m ; \nu=1, \cdots, n)$ are the elements of $(\xi) \times(B)$ and hence $\mathfrak{S}=\mathfrak{S} \times \mathfrak{T}$ is a normal semi-field with $\mathbb{S} \times\left(S^{*}\right.$ as automorphism group.

Let us now assume that $\mathbb{S}$ has a normal divisor $\mathfrak{N}$ such that $\mathbb{B} / \mathfrak{N} \cong \mathfrak{S}_{c}{ }^{*}$, a

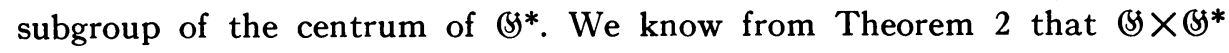

() Deuring, M., Mathematische Annalen, vol. 107 (1932), pp. 140-144. 
$=B_{0} \times\left(S^{*}\right.$ where $\mathscr{S}_{0}$ has the structure given in Theorem 2 . Since the group

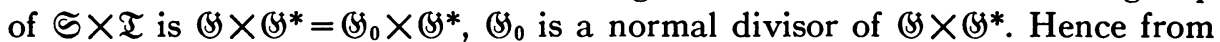
Theorem 1 it follows that $\mathfrak{F}=\mathfrak{S} \times \mathfrak{T}$ contains a normal sub-semi-field $\mathfrak{B}$ of order $n$ whose elements are unaltered by $\$_{0}$ and whose group is $\mathfrak{S}^{*}$. We prove

THEOREM 6. The direct product $\Im=\Im \times \mathfrak{T}=\Im \times \mathfrak{W}$.

For by Theorem $2 \Im$ is the direct product of $\Im\left(\$^{*}\right)$ and $\Im\left(\$_{0}\right)$ since $\left(\mathfrak{S} \times\left(\mathfrak{S}^{*}=\mathfrak{S}_{0} \times \mathfrak{S}^{*}\right.\right.$ and $\mathfrak{\Im}\left(\mathfrak{S}^{*}\right)=\mathfrak{S}$ and $\mathfrak{\Im}\left(\mathfrak{S}_{0}\right)=\mathfrak{W}$. We also see that $\mathfrak{S}_{0}$ can be considered as the group of $\mathfrak{S}$.

For later use we shall construct a basis of $\mathfrak{B}$. Let $\mathbb{S}=\left(G_{1}, G_{2}, \cdots, G_{m}\right)$ have the normal divisor $\mathfrak{N}=\left(n_{1}, n_{2}, \cdots, n_{i}\right)$ consisting of $i$ elements of $(5)$ and let $\mathbb{S}=g_{1} \mathfrak{N}+g_{2} \mathfrak{N}+\cdots+g_{j} \mathfrak{N}, \quad i j=m, \quad \mathbb{F}^{*}=\left(G_{1}^{*}, G_{2}{ }^{*}, \cdots, G_{n}{ }^{*}\right)$ with $\mathfrak{S}_{c}^{*}=\left(c_{1}^{*}, c_{2}^{*}, \cdots, c_{j}^{*}\right)$ a subgroup of the centrum of $\mathcal{S}^{*}$, and $\mathfrak{S} / \mathfrak{N}=\left(g_{1} \mathfrak{N}\right.$, $\left.g_{2} \mathfrak{N}, \cdots, g_{j} \mathfrak{N}\right) \cong \mathfrak{S}_{c}^{*}$. Then every element of $(S)$ is of the form $n_{\nu} g_{\mu}$ and every element of $\mathfrak{S}^{*}$ is a product of a $G^{*}$ and a $c_{\mu}{ }^{*}$. The group $\mathscr{S}_{0}$ consists of elements $S_{0 \nu, \mu}=n_{\nu} g_{\mu} c_{\mu}^{*}(\nu=1, \cdots, i ; \mu=1, \cdots, j)$. For brevity we write $G_{0}=n g c_{0}^{*}$, where $n g$ is in $g \mathfrak{N}$ and $c_{g}^{*}$ is the element of $\mathfrak{S}_{c}^{*}$ corresponding to $g \mathfrak{N}$. Let $u$ and $v$ generate normal bases of $\mathfrak{S}$ and $\mathfrak{T}$ respectively. Then the $n$ elements

$$
w^{G^{*}}=\sum_{\sigma}\left(u^{n_{1}}+u^{n_{2}}+\cdots+u^{n_{i}}\right)^{g} v^{c_{g}^{*} G^{*}}
$$

where $g$ runs over a system of representatives of $(\mathfrak{S} / \mathfrak{N}$, form a normal basis of $\mathfrak{W}$ for all $G^{*}$ of $\mathfrak{S}^{*}$. For every $w^{G^{*}}$ is unaltered by any $H_{0}=h c_{h}^{*}$ of $\mathfrak{S}_{0}$ if $w$ is. But

$$
\begin{aligned}
w^{H_{0}} & =\sum_{g}\left(u^{n_{1}}+u^{n_{2}}+\cdots+u^{n_{i}}\right)^{g n h_{v} c_{g}^{*} c_{h}^{*}} \\
& =\sum_{\sigma}\left(u^{n_{1}}+u^{n_{2}}+\cdots+u^{n_{i}}\right)^{g h_{v} c_{g}^{*} c_{h}^{*}}
\end{aligned}
$$

since $g n=n^{\prime} g$ and $\left(u^{n_{1}}+u^{n_{2}}+\cdots+u^{n_{i}}\right)^{n^{\prime}}=\left(u^{n_{1}}+u^{n_{2}}+\cdots+u^{n_{i}}\right)$. Now $g h$ corresponds to $c_{0}^{*} c_{h}^{*}$ and $c_{0}^{*} c_{h}^{*}$ gives all $j$ elements of $\mathfrak{S}_{c}^{*}$ if $c_{0}^{*}$ runs over all $j$ elements of $\mathfrak{S}_{c}^{*}$. Hence

$$
w^{G_{0}}=w, \quad w^{G^{*}} \text { in } \mathfrak{W} .
$$

The $n$ elements $w^{G^{*}}$ are linearly independent. For the linear combination

$$
\sum_{G^{*}} k_{G^{*}} w^{G^{*}}, \quad k_{G^{*}} \text { in } \Omega,
$$

is a linear combination of the $m n$ elements $u^{G} v^{H^{*}}$ in $\Im$ with the $k_{G^{*}}$ as coefficients, since these elements form a basis of $\Im,(2)$ can only be zero if all $k_{G^{*}}=0$. Thus the $n$ elements $w^{G^{*}}$ of $\mathfrak{W}$ are linearly independent and since $\mathfrak{W}$ has order $n$ they form a normal basis of $\mathfrak{W}$.

Now $\mathfrak{S} / \mathfrak{N}$ and $\mathfrak{S}_{c}^{*}$ are abelian groups. If $g \mathfrak{N} \leftrightarrow c_{0}^{*}$ is an isomorphism between $\mathfrak{S} / \mathfrak{N}$ and $\mathfrak{S}_{c}^{*}$, so is $g \mathfrak{N} \leftrightarrow\left(c_{0}^{*}\right)^{\sigma}$ for an arbitrary integer $\sigma$. For if 
$g \mathfrak{N} \leftrightarrow\left(c_{o}^{*}\right)^{\sigma}, g \mathfrak{R} h \mathfrak{N}=g h \mathfrak{N} \leftrightarrow\left(c_{g}^{*}\right)^{\sigma}\left(c_{h}^{*}\right)^{\sigma}=\left(c_{0}^{*} c_{h}^{*}\right)^{\sigma}$. Each of these isomorphisms yields a $\mathfrak{S}_{0 \sigma}$ and a factorization $\mathfrak{S} \times \mathcal{S}^{*}=\mathfrak{S}_{0 \sigma} \times \mathfrak{S}^{*}$. Consequently $\Im=\mathfrak{S} \times \mathfrak{T}$ $=\Im \times \mathfrak{W}_{\sigma}$, where $\mathfrak{W}_{\sigma}$ is the normal sub-semi-field $\mathfrak{S}\left(\mathfrak{S}_{0 \sigma}\right)$ and has a basis $w_{\sigma}^{G^{*}}=\sum_{\sigma}\left(u^{n_{1}}+\cdots+u^{n_{i}}\right)^{\sigma_{V^{(}}\left(c_{\sigma}^{*}\right)^{\sigma} G^{*}}$ for all $G^{*}$ of $\left(\mathbb{S}^{*}\right.$.

For every $\mathfrak{S}$ and $\mathfrak{T}$ we shall call the set $\mathfrak{W}_{-1}$ defined by $\mathfrak{S} \times \mathfrak{T}=\mathfrak{S} \times \mathfrak{W}_{-1}$ the product of $\mathfrak{S}$ and $\mathfrak{T}$. Then we prove

Lemma 1. The product of $\subseteq$ and $\mathfrak{W}_{-1}$ is $\mathfrak{W}_{-2}$.

For $\mathfrak{S}_{0,-1}$, the group of $\subseteq$, has a normal divisor $\mathfrak{R}$, such that $\mathfrak{H}_{0,-1} / \mathfrak{N}$ $\cong \mathfrak{S}_{c}^{*}, \mathfrak{S}_{0,-1}=g_{1}\left(c_{1}^{*}\right)^{-1} \mathfrak{N}+g_{2}\left(c_{2}^{*}\right)^{-1} \mathfrak{N}+\cdots+g_{j}\left(c_{j}^{*}\right)^{-1} \mathfrak{N}$ and the resulting $\mathfrak{S}_{0,-1}^{\prime}$ $=\left(n g\left(c_{0}^{*}\right)^{-1}\left(c_{0}^{*}\right)^{-1}\right.$, for all $n$ and $\left.g\right)$ is equal to $\mathbb{B}_{0,-2}$ since $n g\left(c_{0}^{*}\right)^{-1}\left(c_{0}^{*}\right)^{-1}$ $=n g\left(c_{0}^{*}\right)^{-2}$. We shall make the induction part of this result later.

5. Normal systems. We now make the

DEFINITION. Let $\subseteq$ and $\widetilde{S}^{\prime}$ be normal semi-fields of order $n$ with respective groups $\left(\mathfrak{S}=(G)\right.$ and $\mathfrak{S}^{\prime}=\left(G^{\prime}\right)$. Then the pairs $\mathfrak{S}$, $\mathbb{S}$ and $\mathfrak{S}^{\prime}$, $\mathfrak{S}^{\prime}$ are called equivalent, if $\mathcal{G} \cong \mathcal{S}^{\prime}$ and if there exists a simple isomorphism

$$
s \leftrightarrow s^{\prime} \quad\left(s \text { in } \mathfrak{S}, s^{\prime} \text { in } \mathfrak{S}^{\prime}\right)
$$

of $\mathfrak{S}$ and $\mathfrak{S}^{\prime}$ such that

$$
\left(s^{G}\right)^{\prime}=s^{\prime G^{\prime}}
$$

for every $s$ of $\mathfrak{S}$.

If the respective pairs $\mathfrak{S}, \mathfrak{B}$ and $\mathfrak{S}^{\prime}, \mathfrak{S}^{\prime} ; \mathfrak{T}, \mathfrak{S}^{*}$ and $\mathfrak{T}^{\prime}$, $\mathfrak{S}^{* \prime}$ are equivalent, the pairs $\mathfrak{S} \times \mathfrak{T}, \mathfrak{S}^{\prime} \times \mathfrak{F}^{*}$ and $\mathfrak{S}^{\prime} \times \mathfrak{T}^{\prime}, \mathfrak{S}^{\prime} \times \mathfrak{S}^{* \prime}$ are equivalent.

The class of all equivalent pairs $\mathfrak{S}, \mathbb{S}$ shall be designated by $(\mathfrak{S},(\mathfrak{S})$ and be called a normal system of degree $n$ with group (\$). Then it follows that if $\left(\mathfrak{S},(\mathfrak{S})=\left(\mathfrak{S}^{\prime}, \mathfrak{S}^{\prime}\right)\right.$ and $\left(\mathfrak{T}, \mathfrak{S}^{*}\right)=\left(\mathfrak{T}^{\prime}, \mathfrak{S}^{* \prime}\right)$, the corresponding systems $\left(\mathfrak{W}_{\sigma}, \mathfrak{S}^{*}\right)$ and $\left(\mathfrak{W}_{\sigma}^{\prime}, \mathfrak{S}^{* \prime}\right)$ of the previous section are equal, if we consider in forming $\mathfrak{W}_{\sigma}^{\prime}$ the normal divisor $\mathfrak{N}^{\prime}$ of $\mathfrak{H}^{\prime}$ which corresponds to $\mathfrak{N}$ of $\mathbb{S}$ in the isomorphism $\mathfrak{B} \cong \$^{\prime}$.

THEOREM 7. The set $\Sigma$ of all normal systems with the same group $\mathbb{B}$, having a normal divisor $\mathfrak{N}$ such that

$$
\text { (S) } / \mathfrak{N} \cong \mathfrak{S}_{c},
$$

$\mathfrak{S}_{c}$ a subgroup of the centrum of $\mathfrak{H}$, is closed with respect to the operation

$$
\left(\mathfrak{S},(\mathfrak{S})\left(\mathfrak{T}, \mathfrak{S}^{*}\right)=\left(\mathfrak{W}_{-1}, \mathfrak{S}^{*}\right)\right.
$$

and

$$
\left(\mathfrak{W}_{-\sigma}, \mathfrak{S}^{*}\right)=(\mathfrak{S}, \mathfrak{S})^{\sigma}(\mathfrak{T}, \mathfrak{S} *) .
$$

The system (ङ, (5), ( a diagonal algebra, is a left unit in $\Sigma$ and every element in $\Sigma$ has finite order. 
For, using our hypothesis of $\$ B$, we can form the product in the given sense, and this product is unique if we take a fixed normal divisor $\mathfrak{N}$ of $\mathfrak{S}$. Note that while the product $\left(\mathfrak{W}_{-1}, \mathfrak{S}^{*}\right)$ is unique there can exist other systems $\left(\mathfrak{S}^{\prime},(\mathfrak{S )})\right.$ such that $\left(\mathfrak{S}^{\prime}, \mathfrak{S}\right)\left(\mathfrak{T}, \mathfrak{S}^{*}\right)=\left(\mathfrak{W}_{-1}, \mathfrak{S}^{*}\right)$. From the structure of $\mathfrak{W}_{-1}$ it is obvious that the product is not necessarily commutative.

We have $\left(\mathfrak{W}_{-1}, \mathfrak{S}^{*}\right)=(\mathfrak{S}, \mathfrak{S})\left(\mathfrak{T}, \mathfrak{S}^{*}\right)$. Assume (4) to be correct for all $\sigma \leqq \tau-1$, therefore $\left(\mathfrak{W}_{-(\tau-1)}\right.$, (ㅑ) $)=\left(\mathfrak{S},(\mathfrak{S})^{r-1}\left(\mathfrak{T}, \mathfrak{B}^{*}\right)\right.$. Then $(\mathfrak{S}, \mathfrak{S})=\left(\mathfrak{S}, \mathfrak{S}^{\prime}\right)$ with $\mathfrak{B S}^{\prime}=\left(n_{0} c_{g}^{*-(\tau-1)}\right)$, and the product of $\mathfrak{S}$ and $\mathfrak{W}_{-(\tau-1)}$ is $\mathfrak{W}_{-\tau}$ from Lemma $1,(\mathfrak{S}, \mathfrak{B})\left(\mathfrak{W}_{-(\tau-1)}, \mathfrak{S}^{*}\right)=\left(\mathfrak{W}_{-r}, \mathfrak{S}^{*}\right)=(\mathfrak{S}, \mathfrak{S}) r\left(\mathfrak{T}, \mathfrak{S}^{*}\right)$.

Let $(\mathbb{E}, \mathfrak{S})\left(\mathfrak{S}, \mathfrak{S}^{*}\right)=\left(\mathfrak{W}_{-1}, \mathfrak{S}^{*}\right)$. Then if $\mathbb{E}=\left(e^{G}\right), \mathfrak{S}=\left(u^{G^{*}}\right), \mathfrak{W}_{-1}=\left(w^{G^{*}}\right)$,

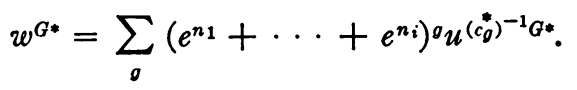

Let $e^{n_{1}}+\cdots+e^{n_{i}}=f$; then $f^{o}$ is a set of $j$ orthogonal idempotents. But $\mathfrak{S}$, ( $)^{*}$

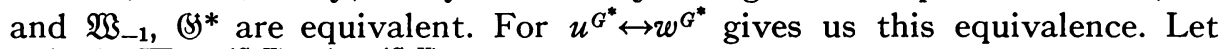
$u^{G^{*}} u^{H^{*}}=\sum_{F^{*}} \rho_{F^{*}}^{(G)} u^{F^{*}}\left(\rho_{F^{*}}^{(G, H)}\right.$ in $\left.\Omega\right)$; then

$$
\begin{aligned}
& w^{G *} w^{H^{*}}=\sum_{o}\left(f^{0}\right) u^{G^{*}\left(c_{0}^{*}\right)^{-1}} \sum_{h}\left(f^{h}\right) u^{H^{*}\left(c_{h}^{*}\right)^{-1}} \\
& =\sum_{g} \sum_{h}\left(f^{0} f^{h}\right) u^{G^{*}\left(c_{g}^{*}\right)^{-1}} u^{H^{*}\left(c_{h}^{*}\right)^{-1}} \\
& =\sum_{g} f^{0}\left(u^{G^{*}} u^{H^{*}}\right)^{\left(c_{g}^{*}\right)^{-1}} \\
& =\sum_{0} f^{0} \sum_{F^{*}} \rho_{F^{*}}^{(G, H)} u^{F^{*}\left(c_{g}^{*}\right)^{-1}} \\
& =\sum_{F^{*}} \rho_{F^{*}}^{(G, H)} \sum_{g}\left(f^{0}\right) u^{F^{*}\left(c_{g}^{*}\right)^{-1}} \\
& =\sum_{F^{*}} \rho_{F^{*}}^{(G, H)} w^{F *}
\end{aligned}
$$

Hence $\left(\mathfrak{S}, \mathfrak{S}^{*}\right)=\left(\mathfrak{W}_{-1}, \mathfrak{S}^{*}\right)$ and $(\mathfrak{S}, \mathfrak{S})\left(\mathfrak{S}, \mathfrak{S}^{*}\right)=\left(\mathfrak{S}, \mathfrak{S}^{*}\right)$.

From (4) we have

$$
\left(\mathfrak{W}_{-j}, \mathfrak{S}^{*}\right)=(\mathfrak{S}, \mathfrak{S})^{j}\left(\mathfrak{T}, \mathfrak{S}^{*}\right) ;
$$

$\mathfrak{W}_{-j}$ consists of all elements of $\mathfrak{S} \times \mathfrak{T}$ unaltered by $n g\left(c_{0}^{*}\right)^{-j}$. But $j$ is the order of $\mathfrak{S}_{c}$ and $\left(c_{0}^{*}\right)^{-i}$ is the unity of $\mathfrak{B}^{*}, \mathfrak{W}_{-j}=\mathfrak{T},\left(\mathfrak{T}, \mathfrak{S}^{*}\right)=(\mathfrak{S}, \mathfrak{G})^{i}\left(\mathfrak{T}, \mathfrak{S}^{*}\right)$. Hence every element has finite order in $\Sigma$. It does not follow, however, that $\left(\mathfrak{S},(\mathfrak{S})^{j}\right.$

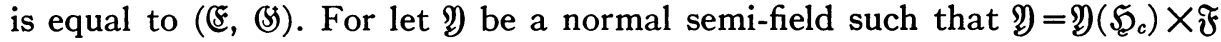
with $\mathfrak{F}$ a diagonal algebra and $\mathfrak{Y}\left(\mathfrak{S}_{c}\right)$ an arbitrary normal semi-field with group $\mathfrak{N}$; then a simple computation shows that $(\mathfrak{V}, \mathfrak{S})\left(\mathfrak{T}, \mathfrak{S}^{*}\right)=\left(\mathfrak{T}, \mathfrak{S}^{*}\right)$ for all $\left(\mathfrak{T}, \mathfrak{S}^{*}\right)$ of $\Sigma$.

Theorem 8. The associative law holds in the set $\Sigma$ of Theorem 7 if and only if

$$
\mathfrak{S}=\mathfrak{N} \times \mathfrak{S}_{c} \text {. }
$$


For let

$$
\left[(\mathfrak{S}, \mathfrak{S})\left(\mathfrak{T}, \mathfrak{S}^{*}\right)\right]\left(\Re, \mathfrak{S}^{* *}\right)=\left(\mathfrak{W}, \mathfrak{S}^{*}\right)\left(\Re, \mathfrak{S}^{* *}\right)=\left(\mathfrak{W}_{0}, \mathfrak{S}^{* *}\right) .
$$

Then $\mathfrak{W}_{0}$ is the normal sub-semi-field of $\mathfrak{S} \times \mathfrak{T} \times \Re$ unaltered by the group $\left(n g\left(c_{g}^{*}\right)^{-1}\right) \times\left(n^{*} g^{*}\left(c_{g^{*}}^{* *}\right)^{-1}\right)$. On the other hand

$$
(\mathfrak{S}, \mathfrak{S})\left[\left(\mathfrak{T}, \mathfrak{S}^{*}\right)\left(\mathfrak{R}, \mathfrak{S}^{* *}\right)\right]=(\mathfrak{S}, \mathfrak{S})\left(\mathfrak{W}_{1}, \mathfrak{S}^{* *}\right)=\left(\mathfrak{W}_{10}, \mathfrak{S}^{* *}\right) \text {. }
$$

$\mathfrak{W}_{10}$ is the normal sub-semi-field of $\mathfrak{S} \times \mathfrak{T} \times \mathfrak{R}$ unaltered by the group $\left(n^{*} g^{*}\left(c_{g^{*}}^{* *}\right)^{-1}\right) \times\left(n g\left(c_{g^{*}}^{* *}\right)^{-1}\right)$.

The associative law holds if $\left(\mathfrak{W}_{0},\left(\mathfrak{S}^{* *}\right)=\left(\mathfrak{W}_{10},\left(\mathfrak{S}^{* *}\right)\right.\right.$, in other words if $\mathfrak{W}_{0}$, $\mathfrak{S}^{* *}$ is equivalent to $\mathfrak{W}_{10}$, $\mathfrak{S}^{* *}$. For this $\mathfrak{W}_{0}$ must be isomorphic to $\mathfrak{W}_{10}$. Let us consider the special case where $\mathfrak{S}, \mathfrak{T}, \mathfrak{R}$ are normal fields whose intersections are $\Omega$. Then $\mathfrak{W}_{0}$ and $\mathfrak{W}_{10}$ are normal fields since they are sub-fields of the field $\mathfrak{S} \times \mathfrak{T} \times \mathfrak{R}$. Then we have $\mathfrak{W}_{0}$ isomorphic to $\mathfrak{W}_{10}$ if and only if the subgroups of $B \times(B) \times(S * *$ whose elements leave their elements unaltered are conjugate subgroups of $\mathbb{B} \times\left(\mathbb{S}^{*} \times\left(\mathbb{S}^{* *}\left({ }^{10}\right)\right.\right.$. But these subgroups are normal divisors of $\mathfrak{S}^{\circ} \times \mathfrak{S}^{*} \times\left(\mathfrak{S}^{* *}\right.$, hence $\mathfrak{W}_{0} \cong \mathfrak{B}_{10}$ if and only if their corresponding groups are equal and $\mathfrak{W}_{0}=\mathfrak{W}_{10}$. The equality of the groups $\left(n g\left(c_{0}^{*}\right)^{-1}\right) \times\left(n^{*} g^{*}\left(c_{\sigma^{*}}^{* *}\right)^{-1}\right)$ and $\left(n g\left(c_{g}^{* *}\right)^{-1}\right) \times\left(n^{*} g^{*}\left(c_{g^{*}}^{* *}\right)^{-1}\right)$ includes, of course, the equivalence of $\mathfrak{B}_{0}$, $\mathfrak{B}^{* *}$ and $\mathfrak{W}_{10}$, (5** in general. Thus the associative law holds in $\Sigma$ if and only if

$$
\left(n g\left(c_{g}^{*}\right)^{-1}\right) \times\left(n^{*} g^{*}\left(c_{g *}^{* *}\right)^{-1}\right)=\left(n g\left(c_{g}^{* *}\right)^{-1}\right) \times\left(n^{*} g^{*}\left(c_{g *}^{* *}\right)^{-1}\right) .
$$

If (7) holds we have $g\left(c_{0}^{*}\right)^{-1}$ in the left member of (7) and

$$
g\left(c_{0}^{*}\right)^{-1}=n g\left(c_{0}^{* *}\right)^{-1} n^{*} h^{*}\left(c_{h^{*}}^{* *}\right)^{-1} .
$$

Hence $n$ and $n^{*}$ are the unities of $\mathfrak{N}$ and $\mathfrak{N}^{*}$ respectively, $\left(c_{h^{*}}^{* *}\right)^{-1}=c_{g}^{* *}$, $h^{*}=g^{*-1}$, and $g\left(c_{g}^{*}\right)^{-1}=g g^{*-1}$ and consequently

$$
c_{g}^{*}=g^{*} \text {. }
$$

But this means $\mathbb{S}=c_{1} \mathfrak{N}+c_{2} \mathfrak{N}+\cdots+c_{j} \mathfrak{N}$. Since the $c_{\nu}$ are in the centrum of $\mathbb{S}$ and $i j=n, i, j, n$ the orders of $\mathfrak{R}, \mathfrak{S}_{c}$, (S) respectively, it follows $\left({ }^{11}\right)$ that $\left(\mathfrak{S}=\mathfrak{N} \times \mathfrak{S}_{c}\right.$.

Our necessary condition (8) is sufficient. For if (8) holds, $\mathfrak{W}_{0}$ in (5) is the subset of $\subseteq \times \mathfrak{T} \times \Re$ unaltered by $\left(n c c^{*-1}\right) \times\left(n^{*} c^{* \prime} c^{* * \prime-1}\right)$ and $\mathfrak{W}_{10}$ the subset unaltered by $\left(n c c^{* *-1}\right) \times\left(n^{*} c^{* \prime} c^{* * \prime-1}\right)$. But $n c c^{*-1} n^{*} c^{* \prime} c^{* * \prime-1}$ $=n c c^{* *-1} n^{*}\left(c^{*-1} c^{* \prime}\right)\left[c^{* *}\left(c^{* * \prime}\right)^{-1}\right]$; hence the groups are equal and therefore $\left(\mathfrak{W}_{0},\left(\mathfrak{S}^{* *}\right)=\left(\mathfrak{W}_{10}, \mathfrak{S}^{* *}\right)\right.$. This proves the theorem.

As an immediate consequence we have the

CoRollary. Let $\mathfrak{S}=\mathfrak{N} \times \mathfrak{S}_{c}$; then $\left(\mathfrak{S},(\mathfrak{S})^{j}\right.$ is a left and right unit of $(\mathfrak{S}, \mathfrak{S})$.

(10) Albert, Modern Higher Algebra, p. 176, Theorem 6.

(11) Ibid., p. 127. 
For the associative law holds and

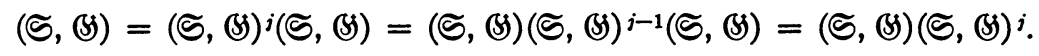

In general there does not exist a right unit for all elements in $\Sigma$. For if $\left(\mathfrak{B}, \mathfrak{S}^{*}\right)$ were a right unit, $(\mathfrak{S}, \mathfrak{B})\left(\mathfrak{B}, \mathfrak{S}^{*}\right)=\left(\mathfrak{S}, \mathfrak{S}^{*}\right)$ for all $(\mathfrak{S}, \mathfrak{B})$, we would have in particular $(\mathfrak{F}, \mathfrak{S})\left(\mathfrak{B}, \mathfrak{S}^{*}\right)=\left(\mathfrak{F}, \mathfrak{S}^{*}\right)$; but $(\mathfrak{E}, \mathfrak{S})\left(\mathfrak{B}, \mathfrak{S}^{*}\right)=\left(\mathfrak{B}, \mathfrak{S}^{*}\right)$; hence

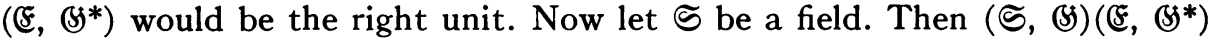
$=\left(\mathfrak{W}_{-1}, \mathfrak{S}^{*}\right)$, and if $\mathfrak{S}=\left(u^{G}\right), \mathfrak{E}=\left(e^{G^{*}}\right), \mathfrak{W}_{-1}=\left(w^{G^{*}}\right)$, then

$$
w^{n^{*} c^{* \prime}}=\sum_{c}\left(u^{n_{1}}+\cdots+u^{n_{i}}\right)^{c} e^{c^{*-1} n^{*} c^{* \prime}}
$$

and

$$
\begin{aligned}
& w w^{n^{*}}=\sum_{c}\left(u^{n_{1}}+\cdots+u^{n_{i}}\right)^{c} e^{c^{*}-1} \sum_{c^{\prime}}\left(u^{n_{1}}+\cdots+u^{n_{i}}\right)^{c^{\prime}} e^{c^{* \prime}-1} n^{*} \\
& =\sum_{c} \sum_{c^{\prime}}\left(u^{n_{1}}+\cdots+u^{n_{i}}\right)^{c}\left(u^{n_{1}}+\cdots+u^{n_{i}}\right)^{c^{\prime}} e^{c^{*}-1} e^{c^{*}-1} n^{*} \\
& =0
\end{aligned}
$$

since every $e^{c^{*}-1} e^{c^{*}-1} n^{*}=0$. Thus $\mathfrak{W}_{-1}$ contains divisors of zero and cannot be equivalent to the field $\mathfrak{S}$; (\&, (\$) is not a right unit-a contradiction.

Let now $\$$ be abelian. Then $B$ is its own centrum and we can consider the factorization $\mathfrak{S} \times \mathfrak{T}=\mathfrak{S} \times \mathfrak{W}_{-1}$ deriving from the isomorphism $\mathfrak{S} \cong\left(\mathbb{S}^{*}\right.$. We do this and have

THEOREM 9. The set $\Sigma$ of all normal systems of order $n$ with the same abelian group $\&$ forms an abelian group with respect to the operation

$$
(\mathfrak{S}, \mathfrak{S})\left(\mathfrak{T}, \mathfrak{S}^{*}\right)=\left(\mathfrak{W}_{-1}, \mathfrak{S}^{*}\right)
$$

and

$$
\left(\mathfrak{W}_{-\sigma}, \mathfrak{S}^{*}\right)=\left(\mathfrak{S},(\mathfrak{S})^{\sigma}\left(\mathfrak{T}, \mathfrak{S}^{*}\right) .\right.
$$

The identity element in $\Sigma$ is the system (\&, (\$) where $\&$ is diagonal and every element of $\Sigma$ has finite order.

We only have to show that the multiplication in $\Sigma$ is commutative, for all the other properties are then immediate consequences of Theorems 7 and 8 . Let $\mathfrak{S}=\left(u^{G}\right), \mathfrak{T}=\left(v^{G^{*}}\right)$. Then $\mathfrak{W}_{-1}=\left(w^{G^{*}}\right)$ with $w=\sum_{G} u^{G} v^{G^{*-1}}$ and from this it follows that $(\mathfrak{S}, \mathfrak{S})\left(\mathfrak{T}, \mathfrak{S}^{*}\right)=\left(\mathfrak{T}, \mathfrak{S}^{*}\right)(\mathfrak{S}, \mathfrak{S})$.

This theorem can also be derived as a consequence of the cyclic case.

Let now $\$$ be cyclic of order $n . S=[S]$ has the identity as normal divisor and $B=[I] \times[S]$ and we can form products of two normal semi-fields with $(B)$ as group. Albert discusses this case $\left.{ }^{12}\right)$. But in case the order $n$ of $B$ is not a prime, $n=i j$, we have a subgroup $\left[S^{i}\right]$ of $(\$)$, which is, of course, a normal divi-

(12) Structure of Algebras, 1939. 
sor of order $j$ of $(\xi)$. Now $\left(\mathfrak{S}=\left[S^{i}\right]+S\left[S^{i}\right]+\cdots+S^{i-1}\left[S^{i}\right]\right.$ and $\mathbb{S} /\left[S^{i}\right] \cong\left[S^{i}\right]$. Consequently we can consider groups $\mathcal{S}_{0,-\sigma}, \sigma=1, \cdots, i-1$, arising from this relation. $\$_{0,-\sigma}$ consists of elements of the form $S^{i \nu} S^{\mu} S^{*-j \sigma \mu}$. It is easily seen that $\left(\mathfrak{S}_{0,-\sigma}\right.$ is a cyclic group of order $n$ generated by $S S^{*-j \sigma}$. Hence $\mathfrak{S H}_{0,-\sigma}$ is the same as the group $\mathfrak{S}_{0,-j \sigma}^{\prime}$ arising from the isomorphism $\mathbb{S}=\mathbb{S} /[I] \cong\left(S^{*}\right.$, and we do not get any new direct factorizations $\mathfrak{S} \times \mathfrak{W}$ by considering $\mathfrak{S}_{0,-\sigma}$.

Note that we considered only factorizations $\mathfrak{S} \times \mathfrak{T}=\mathfrak{S} \times \mathfrak{W}$ which originated by factorizations of the group $\mathfrak{S}^{*} \times \mathcal{S}^{*}=\mathbb{S}_{0} \times \mathcal{S}^{*}$. But there can exist different factorizations of $\mathfrak{S} \times \mathfrak{T}$. Since we are dealing with a generalization of the cyclic case, that is, direct factorizations of $\mathfrak{S} \times \mathfrak{T}$ associated with direct

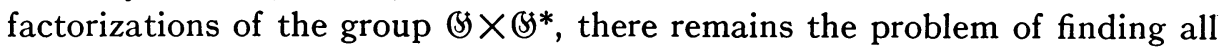
other possible factorizations.

6. Generalized crossed products. Teichmüller defined crossed products of normal semi-fields with their groups similarly to the definition of crossed products of normal fields $\left({ }^{13}\right)$.

Let $\subseteq$ be a normal semi-field of order $n$ and $(S)$ an automorphism group of S. To every two elements $G, H$ of $(\$)$ there shall correspond an element $a_{G, H}$ of $\mathfrak{S}$ such that

$$
a_{F, H} a_{G, F H}=\stackrel{H}{a} a_{G, F} a_{G F, H} .
$$

Furthermore, to every $G$ of $\$$ there corresponds a symbol $x_{G}$ satisfying

$$
\begin{array}{rlr}
s x_{G} & =x_{G} s^{G} \\
x_{G} x_{H} & =x_{G H} a_{G, H} . & \quad(s \text { in } \subseteq),
\end{array}
$$

Then the set

$$
A=(\mathfrak{S}, \mathfrak{S}, a)=\sum_{G} \mathfrak{S} x_{G}
$$

is a normal simple algebra of order $n^{2}$ over $\Re\left({ }^{14}\right)$, and $A=(\Im, ~(5), a)$ is called the crossed product of $\subseteq$ with its group $\mathbb{S}$ and factor set $a$.

Teichmüller proved that if $\mathfrak{S}=\mathfrak{Q} \times \mathbb{E}$,

$$
(\mathfrak{S}, \mathfrak{S}, a) \sim\left(\mathfrak{Q}, \mathfrak{S}, a^{\prime}\right)
$$

with $\mathfrak{S}$ the subgroup of $\mathbb{H}$ belonging to $\mathfrak{Q}$ and $a^{\prime}$ the subset of $a$ corresponding to pairs of elements of $\mathfrak{S}$. Since the crossed product of a normal field with its group is a total matric algebra if the factor set $a=i$, that is, all $a_{G, H}=1$, we have

$$
(\mathfrak{S}, \mathfrak{H}, i) \sim(\mathfrak{Q}, \mathfrak{W}, i) \sim 1
$$

It is easily seen that all theorems which hold for factor sets of crossed

(13) Teichmüller, p. 93.

(14) Ibid., p. 96. 
products of normal fields $\left({ }^{15}\right)$ hold for those of normal semi-fields. In particular those about associated factor sets and that $(\mathfrak{S},(\mathfrak{S}, a) \times(\mathfrak{S}, \mathfrak{( 5 ,} b) \sim(\mathfrak{S},(\mathfrak{S}, a b)$ with the equivalence of algebras, that is, $\mathfrak{A} \sim \mathfrak{B}$ if $\mathfrak{A}=\mathfrak{B} \times \mathfrak{M}$ where $\mathfrak{M}$ is a total matric algebra, and $a b$ is the factor set $c_{G E^{*}, H F^{*}}=a_{G, H} b_{E^{*}, F^{*}}$ hold.

From a theorem by Teichmüller $\left({ }^{16}\right)$ we know that, for two crossed products $A=(\mathfrak{S}, \mathfrak{S}, a)$ and $B=\left(\mathfrak{T}, \mathfrak{S}^{*}, b\right)$ of normal semi-fields $\mathfrak{S}$ and $\mathfrak{T}$ with their respective groups $(\$)$ and $\mathbb{S}^{*}$, the direct product $J=A \times B$ is a crossed product of

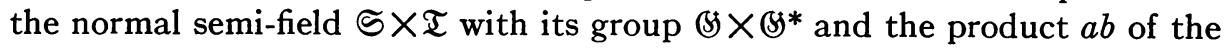
factor sets $a$ and $b$ a factor set:

$$
J=A \times B=(\mathfrak{S}, \mathfrak{S}, a) \times\left(\mathfrak{T},\left(\mathfrak{S}^{*}, b\right)=\left(\mathfrak{S} \times \mathfrak{T}, \mathfrak{S} \times\left(\mathfrak{S}^{*}, a b\right) .\right.\right.
$$

Note that what we have called the factor set $a b=d$ in $\mathfrak{S} \times \mathfrak{T}$ is that factor set consisting of $d_{K, L}=a_{G, H} b_{E^{*}, F^{*}}$ for every $K=G E^{*}, L=H F^{*}$ in $\left.\$\right)^{*} \times()^{*}$, with $G, H$ in (s) and $E^{*}, F^{*}$ in (s).

Let $(S)$ and $\mathbb{B}^{*}$ now be as in Theorem 6 . Then we know from Lemma 1 that $\mathfrak{W}_{-1}$ is contained in $J$. Let us consider the possibility of a factorization of $J$ as direct product of crossed products of $\subseteq$ and $\mathfrak{W}_{-1}$ with their respective groups $\mathfrak{W}_{0}$ and $\mathfrak{B}^{*}$ with $b$ as factor set for $\mathfrak{W}_{-1}$, that is, a factorization

$$
J=A_{1} \times B_{1}=\left(\mathfrak{S}, \mathfrak{S}_{0}, c\right) \times\left(\mathfrak{W}_{-1}, \mathfrak{S} *, b\right) .
$$

From Teichmüller's theorem we know that

$$
\begin{aligned}
\left(\mathfrak{S}, \mathfrak{S}_{0}, c\right) \times\left(\mathfrak{W}_{-1}, \mathfrak{S}^{*}, b\right) & =\left(\mathfrak{S} \times \mathfrak{W}_{-1}, \mathfrak{S}_{0} \times\left(\mathfrak{S}^{*}, c b\right)\right. \\
& =\left(\mathfrak{S} \times \mathfrak{T}, \mathfrak{S} \times \mathfrak{S}^{*}, a b\right) \\
& =(\mathfrak{S}, \mathfrak{S}, a) \times\left(\mathfrak{T}, \mathfrak{S}^{*}, b\right) .
\end{aligned}
$$

Theorem 10. Let $J=(\mathfrak{S}, \mathfrak{S}, a) \times\left(\mathfrak{T}, \mathfrak{S}^{*}, b\right)$ and $\mathfrak{S}, \mathfrak{S}^{*}$ be as in Theorem 6 . Then $J$ has the factorization

$$
J=\left(\mathfrak{S}, \mathfrak{S}_{0}, c\right) \times\left(\mathfrak{W}_{-1},(\mathfrak{S} *, b)\right.
$$

if and only if

(a) the factor set $b$ is in $\mathfrak{T}\left(\mathfrak{S}_{c}^{*}\right)=\mathfrak{T} \cap \mathfrak{W}_{-1}$,

(b) there exist $n$ elements $r_{G_{0}}$ in $\mathfrak{S} \times \mathfrak{T}$, such that

$$
\begin{gathered}
b_{\left(c_{g}^{*}\right)^{-1},\left(c_{t}^{*}\right)^{-1}} R_{G_{0}, H_{0}} \text { in } \subseteq, \\
b_{\left(c_{\theta}^{*}\right)^{-1}, G^{*} r_{G_{0}^{*}}^{G^{*}}}=b_{G^{*},\left(c_{g}^{*}\right)^{-1} r_{G_{0}},}
\end{gathered}
$$

with

$$
c_{G_{0}, H_{0}}=a_{n g, n^{\prime} h} b_{\left(c_{g}^{*}\right)^{-1},\left(c_{k}^{*}\right)^{-1}} R_{G_{0} H_{0}}
$$

where

(15) Albert, A. A., Structure of Algebras, chap. 5.

(16) Teichmüller, p. 100. 


$$
R_{G_{0}, H_{0}}=\frac{{ }_{r_{G_{0}} r_{H_{0}}}^{H_{H_{0}}}}{r_{G_{0} H_{0}}} .
$$

That the factor set $b$ in $\mathfrak{T}$ must consist of elements in $\mathfrak{I} \cap \mathfrak{B}_{-1}$ is trivial since its elements must lie in $\mathfrak{B}_{-1}$ in order that the crossed product $\left(\mathfrak{W}_{-1}, \mathfrak{B}^{*}, b\right)$ has meaning. But $\mathfrak{B}_{-1}$ consists of all elements of $\mathfrak{S} \times \mathfrak{T}$ unaltered by $\$_{0}$. The automorphisms of $\mathbb{S}_{0}$ are products of automorphisms of $B$ with automorphisms of $\mathfrak{S}_{c}^{*}$. Since the elements of $\mathfrak{T}$ are unaltered by $\mathfrak{B}, \mathfrak{T} \cap \mathfrak{W}_{-1}$ consists of all elements of $\mathfrak{I}$ unaltered by $\mathfrak{S}_{c}^{*}$. $\mathfrak{S}_{c}^{*}$ is a normal divisor of $\mathfrak{S}^{*}$, and from Theorem 1 we have $\mathfrak{W}_{-1} \cap \mathfrak{T}=\mathfrak{T}\left(\mathfrak{S}_{c}^{*}\right)$.

To prove that the conditions for the factor set $c$ are necessary let us assume that there exists a factor set $c$ such that (13) holds. Let $\left(\mathcal{S}, \mathfrak{S}_{0}, c\right)=\left(u^{G_{0}} z_{H_{0}}\right)$, $(\mathfrak{S}, \mathfrak{S}, a)=\left(u^{\sigma} x_{H}\right),\left(\mathfrak{T}, \mathfrak{S}^{*}, b\right)=\left(v^{\sigma^{*}} y_{H^{*}}\right)$ where the $u, z ; u, x ; v, y ; a, b, c$ satisfy formulas similar to (9), (10) and (11). Then we have the following equations in $J$

$$
u z_{G_{0}}=z_{G_{0}} u^{G}, \quad w z_{G_{0}}=z_{G_{0}} w .
$$

Now an arbitrary element of $\xi_{0}$ has the form $n g\left(c_{0}^{*}\right)^{-1}$ and we write $n g\left(c_{0}^{*}\right)^{-1}$ $=G_{0}$; then we have in $J$

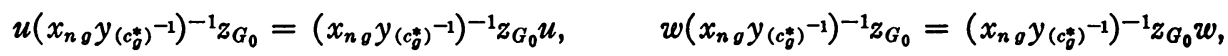
and hence since $v$ is in $\mathfrak{S} \times \mathfrak{W}_{-1}=\mathfrak{S} \times \mathfrak{T}$,

$$
v\left(x_{n g} y_{\left(c_{g}^{*}\right)}^{-1}\right)^{-1} z_{G_{0}}=\left(x_{n} y_{\left(c_{\sigma}^{*}\right)^{-1}}\right)^{-1} z_{G_{0}} v \text {. }
$$

Consequently

$$
\left(x_{n g} y_{\left(c_{g}^{*}\right)}^{-1}\right)^{-1} z_{G_{0}}=r_{G_{0}} \text { in } \mathfrak{S} \times \mathfrak{T},
$$

since the only elements of $J$ commutative with all elements of $\mathfrak{S} \times \mathfrak{T}$ are in $\mathfrak{S} \times \mathfrak{T}$. From (19) it follows that

$$
z_{G_{0}}=\left(x_{n_{0}} y_{\left.\left(c_{g}^{*}\right)^{-1}\right) r_{G_{0}} .}\right.
$$

Now

$$
\begin{aligned}
z_{G_{0}} z_{H_{0}} & =z_{G_{0} H_{0}} c_{G_{0}, H_{0}} \\
& =x_{n g} y_{\left(c_{g}^{*}\right)^{-1} r_{G_{0}} x_{n^{\prime} h} y_{\left(c_{h}^{*}\right)^{-1} r_{H_{0}}}} \\
& =x_{n g n^{\prime} h} y_{\left(c_{g}^{*}\right)^{-1}\left(c_{h}^{*}\right)^{-1}} a_{n g, n^{\prime} h} b_{\left(c_{g}^{*}\right)^{-1},\left(c_{h}^{*}\right)^{-1} r_{G_{0}} r_{H_{0}}} \\
& =x_{n g n^{\prime} h} y_{\left(c_{g}^{*}\right)^{-1}\left(c_{h}^{*}\right)^{-1} r_{G_{0} H_{0}} c_{G_{0}, H_{0}} .}
\end{aligned}
$$

Hence we get (16)

$$
\begin{aligned}
c_{G_{0}, H_{0}} & =a_{n g, n^{\prime} h} b_{\left(c_{g}^{*}\right)^{-1},\left(c_{h}^{*}\right)^{-1}} \frac{r_{G_{0} r_{H_{0}}}^{H_{0}}}{r_{G_{0} H_{0}}} \\
& =a_{n g, n^{\prime} h} b_{\left(c_{g}^{*}\right)^{-1},\left(c_{h}^{*}\right)^{-1}} R_{G_{0}, H_{0}} .
\end{aligned}
$$


The fraction $R_{G_{0}, H_{0}}$ designates merely the result of a transformation of the basis in $J$. The $c$ 's form a factor set since we can derive them by a transformation of a basis of $J$. But the sets $c$ and $a$ are in $\subseteq$ and we have (14)

$$
b_{\left(c_{0}^{*}\right)^{-1},\left(c_{n}^{*}\right)^{-1}} R_{G_{0}, H_{0}} \text { in } \subseteq \quad \text { for all } G_{0}, H_{0} .
$$

In addition, since we want to form the direct product $\left(\mathfrak{S}, \mathfrak{S}_{0}, c\right) \times\left(\mathfrak{B}_{-1}, \mathfrak{S}^{*}, b\right)$ we must have

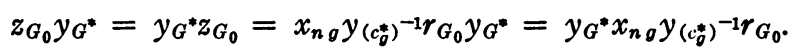

The $x$ and $y$ are commutative and consequently we have (15)

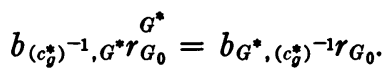

The conditions are sufficient. For with $c$ as in (16) we have $\left(\mathfrak{S}, \mathfrak{S}_{0}, c\right)$ $\times\left(\mathfrak{W}_{-1},\left(\mathfrak{S}^{*}, b\right)=\left(\mathfrak{S} \times \mathfrak{W}_{-1}, \mathfrak{S}_{0} \times\left(\mathfrak{S}^{*}, c b\right)\right.\right.$ and $c b$ is a factor set in $S \times \mathfrak{B}_{-1}$ $=\mathfrak{S} \times \mathfrak{T}$ associated with $a b$ and therefore $\left(\mathfrak{S}, \mathfrak{S}_{0}, c\right) \times\left(\mathfrak{W}_{-1}, \mathfrak{S}^{*}, b\right)$ $=\left(S \times \mathfrak{W}_{-1}, \mathfrak{S}_{0} \times \mathfrak{S}^{*}, c b\right)=\left(\mathfrak{S} \times \mathfrak{T}, \mathfrak{S} \times(\mathfrak{S} *, a b)=(\mathfrak{S}, \mathfrak{G}, a) \times\left(\mathfrak{T}, \mathfrak{S}^{*}, b\right)\right.$. This proves Theorem 10 .

Note that the factor set $c$ consists of elements which are products of elements of $a$ with elements of that part of $b$ which corresponds to pairs of automorphisms of $\mathfrak{S}_{c}^{*}$. By a change of the basis of $J$ these products are multiplied by $R_{G_{0}, H_{0}}$ such that $c$ is in $\mathfrak{S}$.

In case $\mathfrak{S}^{*}=\mathfrak{N}^{* \prime} \times \mathfrak{S}_{c}^{*}$ we have $\mathfrak{T}=\mathfrak{T}\left(\mathfrak{N}^{* \prime}\right) \times \mathfrak{T}\left(\mathfrak{W}_{c}^{*}\right)$. Then the factor set $b$ may be such that $\left(\mathfrak{T}, \mathfrak{S S}^{*}, b\right)=\left(\mathfrak{T}\left(\mathfrak{R}^{* \prime}\right), \mathfrak{H}_{c}^{*}, b^{\prime}\right) \times\left(\mathfrak{T}\left(\mathfrak{S}_{c}^{*}\right), \mathfrak{N}^{* \prime}, b^{\prime \prime}\right)$. But if the factor set $b$ satisfies the first condition, the $b^{\prime}$ are in $\Omega$, since they are in $\mathfrak{T}\left(\mathfrak{R}^{* \prime}\right)$ and $\mathfrak{T}\left(\mathfrak{S}_{c}^{*}\right)$ and their intersection is $\Re$. This gives

THEOREM 11. If $\mathbb{H}=\mathfrak{N}^{* \prime} \times \mathfrak{S}_{c}^{*}, \mathfrak{H} / \mathfrak{N} \cong \mathfrak{S}_{c}^{*}$ is as in Theorem 6 , the factor set $b$ is in $\mathfrak{T}\left(\mathfrak{S}_{c}^{*}\right)$, and $\left(\mathfrak{T}, \mathfrak{S}^{*}, b\right)=\left(\mathfrak{I}\left(\mathfrak{N}^{* \prime}\right), \mathfrak{W}_{c}^{*}, b^{\prime}\right) \times\left(\mathfrak{I}\left(\mathfrak{W}_{c}^{*}\right), \mathfrak{N}^{* \prime}, b^{\prime \prime}\right)$, then

$$
J=(\mathfrak{S}, \mathfrak{S}, a) \times\left(\mathfrak{T}, \mathfrak{S}^{*}, b\right)=\left(\mathfrak{S}, \mathfrak{S}_{0}, c\right) \times\left(\mathfrak{W}_{-1}, \mathfrak{S}^{*}, b\right)
$$

with

$$
c_{G_{0} H_{0}}=a_{n g, n^{\prime} h} b_{\left(c_{g}^{*}\right)^{-1},\left(c_{h}^{*}\right)^{-1}} .
$$

We can consider the crossed product $(\Im, \Im, a)$ as defined by the normal system $\mathfrak{A}=(\mathfrak{S}, \mathfrak{S})$, if the factor set $a$ is in the intersection of all $\mathfrak{S}$ in $\mathfrak{A}$. Now we know from Theorem 8 that if $\mathfrak{S}=\left(u^{G}, \nu=1, \cdots, n\right)$ with group $\mathbb{S}$ having normal divisor $\mathfrak{N}$ such that $\mathbb{H} / \mathfrak{N} \cong \mathfrak{S}_{c}$, a subgroup of the centrum of $\mathbb{H}$, and $\mathfrak{S}^{*}=\left(v^{G_{v}^{*}}\right), v=\sum_{0} f^{g} u^{\left(c_{*}^{*}\right)-1}, f^{v}$ a set of $j$ orthogonal idempotents, then the pairs

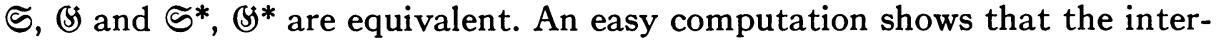
section $\mathfrak{S} \cap \mathfrak{S}^{*}$ is $\mathfrak{S}\left(\mathfrak{S}_{c}\right)$, the sub-semi-field of $\mathfrak{S}$ unaltered by $\mathfrak{S}_{c}$. Thus it is at least necessary that the factor set $a$ is in $\mathfrak{S}\left(\mathfrak{S}_{c}\right)$.

It follows that if $\mathbb{B}$ is abelian the intersection of all $\mathfrak{S}$ in $\mathfrak{A}$ must be $\mathfrak{S}(\$)$ and is therefore $\Omega$. 
In this fashion we write $(\mathfrak{A}, a)$ with $\mathfrak{A}$ a normal system $(\mathfrak{S}, \mathfrak{S})$ and $a$ a factor set in the intersection of all $\mathfrak{S}$ in $\mathfrak{A}$. We say for brevity that $a$ is in $\mathfrak{A}$. Then as a consequence of Theorem 10 we have

THEOREM 12. Let $\mathfrak{A}$ and $\mathfrak{B}$ be normal systems with the same group $\$$ S having the property of Theorem 7. Suppose that $a$ and $b$ are respective factor sets in $\mathfrak{A}$ and $\mathfrak{B}$ such that

(a) the factor set $b$ is in $\mathscr{B}$ and $\mathfrak{A P}$,

(b) there exist $n$ elements $r_{G_{0}}$ in $\mathfrak{A B}$, such that

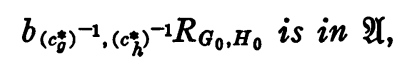

and

$$
b_{\left(c_{g}^{*}\right)^{-1}, G^{*} r_{G_{0}}^{G^{*}}}=b_{G^{*},\left(c_{g}^{*}\right)^{-1} r_{G_{0}},}
$$

with the factor set $c$ as in (16).

Then

$$
(\mathfrak{A}, a) \times(\mathfrak{B}, b)=(\mathfrak{A}, c) \times(\mathfrak{Y} \mathfrak{B}, \mathfrak{Q} b) .
$$

COROLlaRy I. If the factor set $b$ is in the reference field, and

$$
b_{G^{*},\left(c_{g}^{*}\right)^{-1}}=b_{\left(c_{g}^{*}\right)^{-1}, G^{*}}
$$

then $(\mathfrak{A}, a) \times(\mathfrak{B}, b)=(\mathfrak{A}, c) \times(\mathfrak{A} \mathscr{B}, b)$ with $c=\left\{c_{G_{0}, H_{0}}\right\}$,

$$
c_{G_{0}, H_{0}}=a_{n g, n^{\prime} h} b_{\left(c_{g}^{*}\right)^{-1},\left(c_{h}^{*}\right)^{-1}} \text {. }
$$

Corollary II. Let

$$
a_{G, c_{b}}=a_{c_{\theta}, G}
$$

Then

with $c=\left\{c_{G_{0}, H_{0}}\right\}$.

$$
(\mathfrak{A}, a)^{2}=(\mathfrak{A}, c) \times\left(\mathfrak{U}^{2}, a\right)
$$

We now discuss the case where $B$ is abelian. Then we saw that in order to form a crossed product of the system $\mathfrak{A}$ with its group $(B)$ the factor set must be in $\Omega$. We then have

Theorem 13. Let $\mathfrak{A}$ and $\mathfrak{B}$ be abelian systems with group $\mathfrak{B}, a$ and $b$ two factor sets in the reference field, and

$$
b_{G^{*}, H^{*}}=b_{H^{*}, G^{*}}
$$

then $(\mathfrak{A}, a) \times(\mathfrak{B}, b)=\left(\mathfrak{A}, a b^{-1}\right) \times(\mathfrak{A P}, b)$.

This follows from Theorem 12, for the three conditions are satisfied with $r_{G_{0}}=1$.

As a consequence we have 
THEOREM 14. If $\mathfrak{A}$ is an abelian system and

$$
a_{G, H}=a_{H, G},
$$

then

$$
(\mathfrak{A}, a)^{\sigma} \sim\left(\mathfrak{A}^{\sigma}, a\right) .
$$

For from Theorem 14 we have $(\mathfrak{A}, a)^{2}=\left(\mathfrak{A}, a a^{-1}\right) \times\left(\mathfrak{A}^{2}, a\right)$ with $a_{G, H} a_{\overline{G, H}}^{-1}=1$ and hence ( $\left(\mathfrak{A}, a a^{-1}\right)$ is a total matric algebra; this is (23) for $\sigma=2$. In general (23) follows then by repeated use of this argument.

If the group $\mathfrak{B}$ of $\mathfrak{A}$ is abelian and the direct product of not more than two cyclic groups we can show that the crossed product $(\mathfrak{A}, a)$ is the direct product of two cyclic algebras. It is not known whether a similar statement can be made for the case where the group of $\mathfrak{A}$ is the direct product of more than two cyclic factors unless (22) holds, and hence that hypothesis seems to be necessary in order that a result of the type given above may be obtained.

University of Chicago,

Chicago, Ill. 